\title{
Chronic stress enhances progression of periodontitis via $\alpha 1$-adrenergic signaling: a potential target for periodontal disease therapy
}

\author{
Huaixiu $\mathrm{Lu}^{1}$, Minguang $\mathrm{Xu}^{2}$, Feng Wang ${ }^{1}$, Shisen $\mathrm{Liu}^{1}$, Jing Gu${ }^{1}$ and Songshan $\operatorname{Lin}^{1}$
}

This study assessed the roles of chronic stress (CS) in the stimulation of the sympathetic nervous system and explored the underlying mechanisms of periodontitis. Using an animal model of periodontitis and CS, the expression of tyrosine hydroxylase $(\mathrm{TH})$ and the protein levels of the $\alpha 1$-adrenergic receptor ( $\alpha 1$-AR) and $\beta 2$-adrenergic receptor ( $\beta 2$-AR) were assessed. Furthermore, human periodontal ligament fibroblasts (HPDLFs) were stimulated with lipopolysaccharide (LPS) to mimic the process of inflammation. The proliferation of the HPDLFs and the expression of $\alpha 1-A R$ and $\beta 2-A R$ were assessed. The

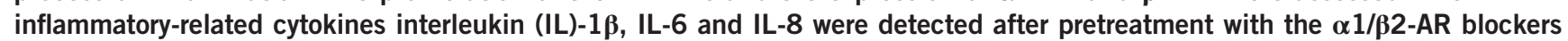
phentolamine/propranolol, both in vitro and in vivo. Results show that periodontitis under CS conditions enhanced the expression of TH, $\alpha 1-A R$ and $\beta 2$-AR. Phentolamine significantly reduced the inflammatory cytokine levels. Furthermore, we observed a marked decrease in HPDLF proliferation and the increased expression of $\alpha 1$-ARfollowing LPS pretreatment. Pretreatment with phentolamine dramatically ameliorated LPS-inhibited cell proliferation. In addition, the blocking of $\alpha 1$-ARsignaling also hindered the upregulation of the inflammatory-related cytokines IL-1ß, IL-6 and IL-8. These results suggest that CS can significantly enhance the pathological progression of periodontitis by an $\alpha 1$-adrenergic signaling-mediated inflammatory response. We have identified a potential therapeutic target for the treatment of periodontal disease, particularly in those patients suffering from concurrent CS.

Experimental \& Molecular Medicine (2014) 46, e118; doi:10.1038/emm.2014.65; published online 17 October 2014

\section{INTRODUCTION}

Periodontitis is a multifactorial disease associated with the destruction of the supporting structures of the teeth, such as the periodontal ligaments and alveolar bones, which affects millions of people each year. ${ }^{1}$ Epidemiologic studies from many regions of the world have demonstrated that periodontitis typically affects individuals in different manners, and only $7-15 \%$ of the dentate adult population suffers from this destructive periodontal disease. ${ }^{2}$ Although bacteria have been identified as the etiologic agents of periodontal disease, ${ }^{3}$ there are several other factors that have important roles in its occurrence; for example, there is strong evidence that destructive processes occur as part of the host inflammatory response and are responsible for the majority of the hard and soft tissue breakdown, leading to the clinical signs of periodontitis. ${ }^{4}$

There are many environmental risk factors that have been associated with the inflammatory response, such as stress, depression and anxiety. Chronic stress (CS) is an organic response involving increased sympathetic outflow and the activation of the hypothalamic-pituitary-adrenal axis as a result of emotional pressure suffered for a prolonged period, over which an individual perceives they have no control. ${ }^{5}$ It involves an endocrine system response during which a release of corticosteroids occurs. Although the immediate effects of stress hormones are beneficial in particular situations, long-term exposure to stress leads to long-lasting sympathetic activity and high levels of the associated hormones, which suppress the immune system, ${ }^{6}$ causing the acceleration of the process of periodontitis. The notion that CS fosters disease by activating the hypothalamic-pituitary-adrenal axis has featured prominently in many hypotheses. Over the past decade, increasing evidence has emerged from epidemiologic studies relating periodontitis to stress, depression and negative life events. ${ }^{7}$ However, the associated underlying mechanisms are unclear.

When CS-triggered signaling reaches the ventromedial nucleus of the hypothalamus from the locus coeruleus, the

${ }^{1}$ Department of Stomatology, Navy General Hospital, Beijing, China and ${ }^{2}$ Department of Medical Engineering, The Second Artillery General Hospital of PLA, Beijing, China

Correspondence: Dr H Lu, Department of Stomatology, Navy General Hospital, Beijing 100048, China.

E-mail: huaixiulu@163.com

Received 26 May 2014; revised 1 August 2014; accepted 13 August 2014 
sympathetic system can be activated, resulting in the release of catecholamine neurotransmitters. ${ }^{8}$ These neurotransmitters can be identified by their tyrosine hydroxylase (TH)-positive statuses. Because $\mathrm{TH}$ is the enzyme responsible for catalyzing the conversion of catecholamine into norepinephrine (noradrenaline) and epinephrine (adrenaline), ${ }^{9}$ the resulting adrenaline combines with its corresponding receptors and causes further intracellular signal transduction.

The adrenergic receptors (ARs) belong to a class of $G$ protein-coupled receptors that are targets of the catecholamines, particularly norepinephrine (noradrenaline) and epinephrine (adrenaline). ${ }^{10}$ Many cells possess these receptors, and the binding of a catecholamine to its receptor will generally stimulate the sympathetic nervous system. There are two main groups of ARs, $\alpha$ and $\beta$, and several subtypes. ${ }^{11}$ Of these ARs, $\alpha 1$-adrenergic receptor $(\alpha 1-\mathrm{AR})$ and $\beta 2$-receptor $(\beta 2-\mathrm{AR})$ are generally considered to be involved in brain-immune communication. ${ }^{12,13}$ Although a previous study has reported the effects of CS on the progression of periodontitis, ${ }^{14}$ the associated mechanisms remain unclear.

The present study was designed to assess the roles of CS in the stimulation of the sympathetic nervous system and in the progression of periodontitis. Furthermore, the underlying mechanisms of periodontitis were also investigated.

\section{MATERIALS AND METHODS}

\section{Antibodies and reagents}

Unless otherwise mentioned, all reagents were purchased from Gibco (Grand Island, NY, USA). Stromal cell basal medium was purchased from Abcam (Cambridge, MA, USA). Phentolamine and propranolol ( $\alpha 1$-, $\beta 2$-adrenergic receptor ( $\beta 2$-AR) blockers) were obtained from Calbiochem (San Diego, CA, USA). Rabbit anti-TH polyclonal antibodies were purchased from Santa Cruz Biotechnology (Santa Cruz, CA, USA). Anti- $\beta$-actin antibodies were obtained from Upstate Biotechnology, Inc. (Lake Placid, NY, USA). Rabbit anti- $\alpha 1-$ AR antibodies were purchased from Abcam. Anti- $\beta 2-A R$ antibodies were obtained from Sigma (St Louis, MO, USA). Monoclonal antibodies against vimentin and keratin were obtained from Cell Signaling Technology (Beverly, MA, USA). Horseradish peroxidaseconjugated goat anti-mouse and anti-rabbit antibodies were purchased from DakoCytomation (Glostrup, Denmark). Lipopolysaccharide (LPS) was purchased from Sigma.

\section{Animals}

Thirty male Wistar rats (Charles River Laboratories, Wilmington, MA, USA) with an average initial weight of $\sim 180 \mathrm{~g}$ were selected. The animals were acclimatized to the housing conditions over the following 4 weeks at $20-24{ }^{\circ} \mathrm{C}$ and $50-70 \%$ relative humidity with a 12-h light/dark cycle. Food and water were available ad libitum, except for those times when the CS was applied. The animals were assigned randomly to three experimental groups after weight stratification as follows: the control group (G1), ligature-induced periodontal disease group (G2) and CS-associated periodontal disease group (G3). Each group contained 10 rats.

\section{Experimental periodontal disease}

All procedures were in agreement with the standards for the care of laboratory animals and were approved by the Medical Ethics Committee of the National Institutes of Health.
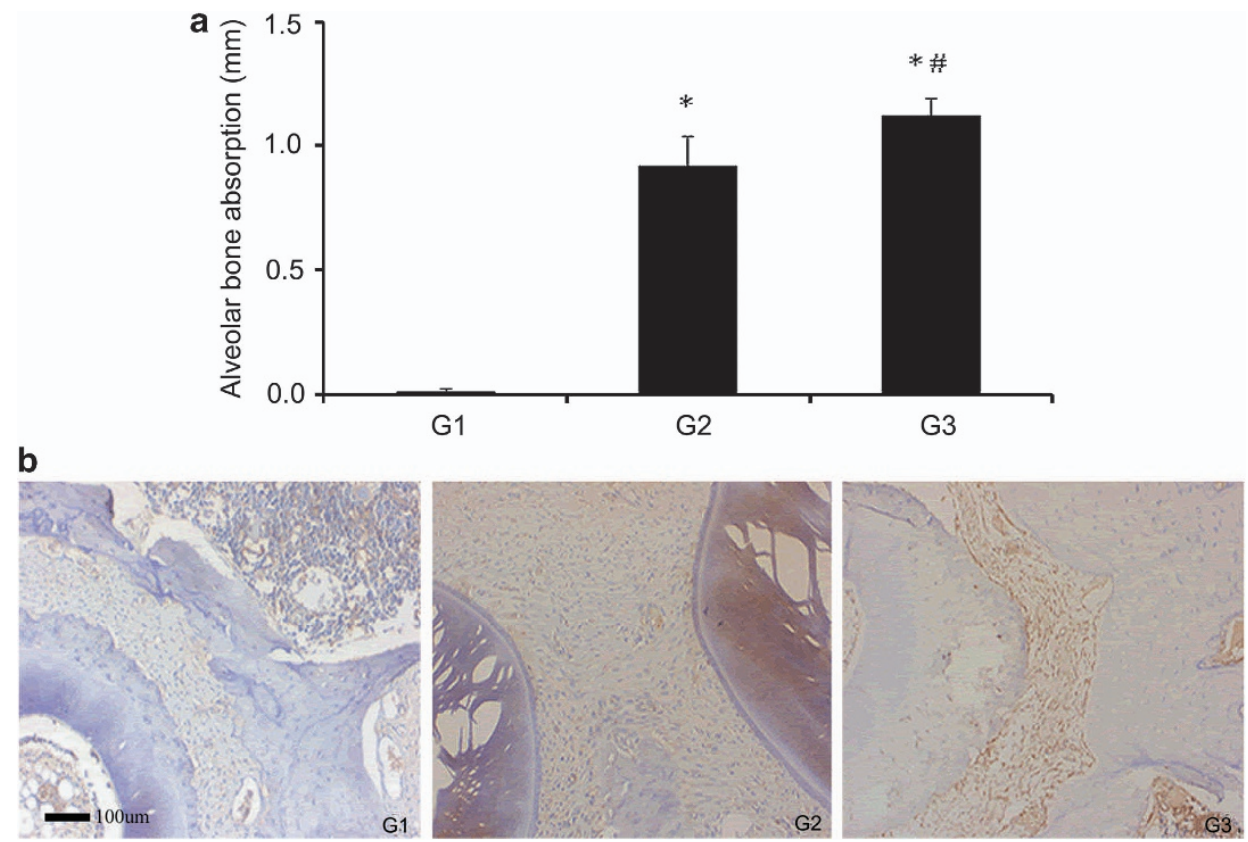

Figure 1 Alveolar bone absorption and the expression of TH in the periodontal tissue. After applying the ligatures for 30 days, alveolar bone absorption around the maxillary first molars was detected, and G3 was found to be more severely affected than G2 (a). TH levels in local tissues were monitored by immunocytochemistry. The hyperchromatic areas in the periodontal ligament cells and the macrophage cytoplasm show the increased expression of TH resulting from the CS treatment (b). *compared with G1 P<0.01, \#compared with G2 $P<0.05$. Scale bar $=100 \mu \mathrm{m}$. 
The animals in the control group were submitted to a sham procedure, whereas the other animals were administered general anesthesia using a $0.5 \mathrm{ml} \mathrm{kg}^{-1}$ intramuscular administration of ketamine hydrochloride (Ketanest; Parke-Davis, Berlin, Germany), and then, the maxillary first molars of each animal received a $0.20-\mathrm{mm}$ nylon thread ligature (Ethicon, Johnson \& Johnson, São Paulo, SP, Brazil) around the neck of the tooth in the dental gingival area to induce experimental periodontal disease as described by Koide et al. ${ }^{15}$ The ligatures were kept in place during the entire experimental period, and after the operation, the G2 and G3 rats received wet food and a syrup drink ( $100 \mathrm{ml}$ water with $10 \mathrm{~g}$ sugar). At the end of the experiment, the marginal gingival tissues containing the alveolar bones and surrounding soft tissues around the maxillary first molars were harvested, and the areas of bone lost were determined.

\section{CS induction}

CS was applied daily to the G3 rats from 8:30 to $10: 30$ am or from 14:30 to $17: 30 \mathrm{pm}$ from the 4 th day after the induction of periodontitis according to Willner ${ }^{16}$ with slight modifications. Each week of the 28-day CS regimen consisted of the following stresses: two periods of food or of water deprivation; two periods of $45^{\circ}$ cage tilt; two periods of cage soiling ( $250 \mathrm{ml}$ water in sawdust bedding); two periods of feet shocking $(5 \mathrm{~min})$; two periods of $4{ }^{\circ} \mathrm{C}$ or $45^{\circ} \mathrm{C}$ swimming ( $5 \mathrm{~min}$ ); two periods of tail clamping ( $1 \mathrm{~min}$ ); two periods of binding; and two periods of no stress. All of these stresses were applied randomly. This model has been shown to induce CS, as demonstrated by neuroendocrine activation, weight loss and anxiety-like behaviors. ${ }^{17}$

\section{Human periodontal ligament fibroblasts (HPDLFs) isolation, culture and characterization}

HPDLFs were isolated from the middle third regions of the roots of the periodontally healthy teeth of adolescents for orthodontic reasons at the PLA Navy General Hospital of Dentistry with the donors' informed consent. The cells were cultured in stromal cell basal medium supplemented with growth factors (basic fibroblast growth factor (bFGF), insulin), $10 \%$ fetal bovine serum and gentamicin/ amphotericin-B at $37^{\circ} \mathrm{C}$ in humidified air with $5 \% \mathrm{CO}_{2}$ (Life Technologies, Baltimore, MD, USA). P4 of the HPDLFs (with an initial density of $5 \times 10^{6}$ cells $\mathrm{ml}^{-1}$ ) was observed using inverted microscopy. The viabilities, proliferation and functioning of the
HPDLFs were characterized, and histological examinations were performed with light microscopy and scanning electron microscopy 15 days after cell seeding. Only those cells that stained positive for vimentin and negative for cytokeratin were identified as HPDLFs.

\section{Immunocytochemistry}

The tissues from the maxilla were first perfused with PBS and then with $4 \%$ paraformaldehyde for $48 \mathrm{~h}$ under physiological pressure before being decalcified in trichloroacetic acid until complete decalcification was confirmed radiographically. After dehydration in graded alcohol, the samples were embedded in paraffin and cut into $6-\mu \mathrm{m}$ serial sections. The sections were incubated overnight at $4{ }^{\circ} \mathrm{C}$ with anti-TH primary antibodies (1:200) and were thereafter processed for $2 \mathrm{~h}$ with horseradish peroxidase-conjugated secondary antibodies. The reactions were visualized with the $\mathrm{ABC}$ complex (Vectastain), and the representative fields were photographed using a Photometrix CoolSNAP Fx camera (Photometrix, Roper scientific, Tucson, AZ) mounted onto a NIKON Eclipse TE 200 UV microscope.

\section{Western blotting analysis}

The periodontal tissue blocks were homogenized and lysed with RIPA lysis buffer (100 mm NaCl, 50 mm Tris- $\mathrm{HCl}, \mathrm{pH} 7.5,1 \%$ Triton X-100, $1 \mathrm{~mm}$ EDTA, $10 \mathrm{~mm}$ beta-glycerophosphate, and $2 \mathrm{~mm}$ sodium vanadate and protease inhibitor). Protein concentrations were measured using the micro-BCA protein assay (Pierce, Rockford, IL, USA). A total of $40 \mathrm{mg}$ of protein per lane was separated by $10 \%$ sodium dodecyl sulfate polyacrylamide gel electrophoresis and electroblotted onto a polyvinylidenedifluoride membrane using a semi-dry trans-blot apparatus. Then, the polyvinylidenedifluoride membrane was blocked by incubation with $5 \%$ nonfat milk in tris buffer saline tween 20 buffer at room temperature for $1 \mathrm{~h}$. The polyvinylidenedifluoride membrane was incubated with anti- $\alpha 1-\mathrm{AR}$, anti- $\beta 2$-AR or anti- $\beta$-actin antibodies for $1 \mathrm{~h}$ at room temperature. Following three washes with tris buffer saline tween 20 buffer, horseradish peroxidase-conjugated secondary antibodies were introduced, and enhanced chemiluminescence (ECL, Amersham Pharmacia, NJ, USA) was used for the detections.

\section{Enzyme-linked immunosorbent assay}

The periodontal tissues were treated with a superfusion system; after stimulation with different inducing factors (LPS, phentolamine or
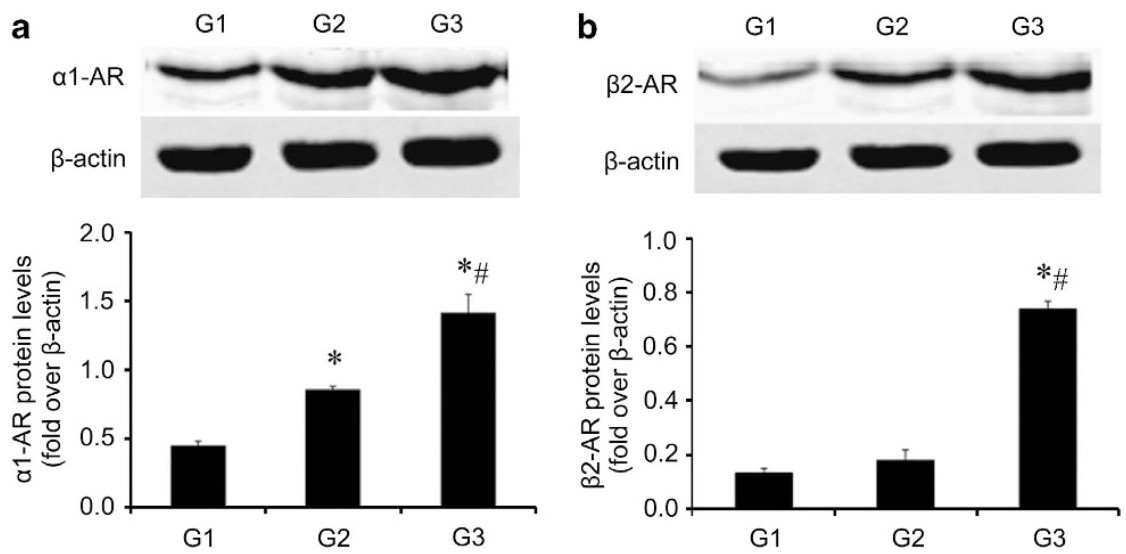

Figure 2 CS exposure increased the expression of the adrenergic receptor protein. G3 was treated with a series of unexpected chronic stresses for 4 weeks, and then, the protein levels of $\alpha 1-A R(a)$, and $\beta 2-A R$ (b) were analyzed by western blotting. ${ }^{*}$ compared with G1 $P<0.01$, " compared with G2 $P<0.01$. 
propranolol) for $4 \mathrm{~h}$, the supernatants were collected. The inflammatory-related cytokines interleukin (IL)-1 $\beta$, IL-6 and IL- 8 were assayed using an enzyme-linked immunosorbent assay kit (Abcam) following the manufacturer's recommendations.

\section{3-(4,5-dimethyl-2-thiazolyl)-2,5-diphenyl-2H-tetrazolium bromide assay}

The HPDLFs were seeded into 96-well culture plates. After 15 days, the cells were treated with the $\alpha / \beta$-AR blockers phentolamine/ propranolol prior to their exposure to $0,5,10$ and $20 \mathrm{ng} \mathrm{ml}^{-1}$ LPS. The cells were incubated for $24 \mathrm{~h}$ or for $48 \mathrm{~h}$ at $37^{\circ} \mathrm{C}$ and $5 \% \mathrm{CO}_{2}$, and the culture medium was then replaced with fresh medium containing $5 \mathrm{ng} \mathrm{ml}^{-1}$ 3-(4,5-dimethyl-2-thiazolyl)-2,5-diphenyl-2Htetrazolium bromide, in which the cells were incubated for an additional $4 \mathrm{~h}$. Finally, the formazan crystals were dissolved in an SDS-HCl solution, and the optical densities were determined with a spectrophotometer at $\lambda 490 \mathrm{~nm}$.

\section{Data analysis}

The results are presented as the mean \pm standard deviation (s.d.). Statistical analyses were performed with a paired or an unpaired Student's $t$-test for the direct two-group comparisons and the TukeyKramer test after a significant one-way analysis of variance $F$-test for the multiple-group comparisons. Differences were considered statistically significant at $P<0.05$.

\section{RESULTS}

\section{Alveolar bone absorption}

Alveolar bone absorption analysis of the furcation region demonstrated significant differences between G2, G3 and G1 (G2 versus G1, $P<0.01$; G3 versus $\mathrm{G} 1, P<0.01$; $\mathrm{G} 3$ versus $\mathrm{G} 2$, $P<0.05)$. Ligature placement resulted in significant bone loss in the region of the first maxillary molar $(P<0.01)$ compared with the non-ligated sites. In addition, CS significantly increased the extent of periodontitis at the ligated sites $(P<0.05)$ compared with that in the non-CS-exposed rats (Figure 1a). These findings suggested that CS can promote the progression of periodontitis.

Enhanced expression of TH in periodontitis following CS The expression of $\mathrm{TH}$ in the gingival tissues around the ligated and non-ligated teeth in the G1, G2 and G3 rats was detected by immunocytochemistry. TH is the enzyme responsible for catalyzing the conversion of the amino acid L-tyrosine to L-3,4dihydroxyphenylalanine, ${ }^{18,19}$ which is a precursor of the important neurotransmitters norepinephrine (noradrenaline) and epinephrine (adrenaline), and it catalyzes the rate-limiting step in the synthesis of catecholamines.

The increased expression of TH in the periodontal ligament cells and in the cytoplasm of macrophages in G3 (Figure 1b) suggested that CS was able to promote sympathetic activity and stimulate the release of catecholamine neurotransmitters, which negatively affected the gingival tissues.

\section{Elevated expression of AR protein was confirmed in} periodontitis

The ARs, particularly norepinephrine and epinephrine, are targets of catecholamines. ${ }^{20}$ To clarify the manner by which these stress-related biobehavioral factors accelerate the progression of periodontitis, a further analysis of the AR levels was performed. The $\alpha 1-\mathrm{AR}$ and $\beta 2$-receptor ( $\beta 2-\mathrm{AR})$ are generally considered to be involved in brain-immune communication.
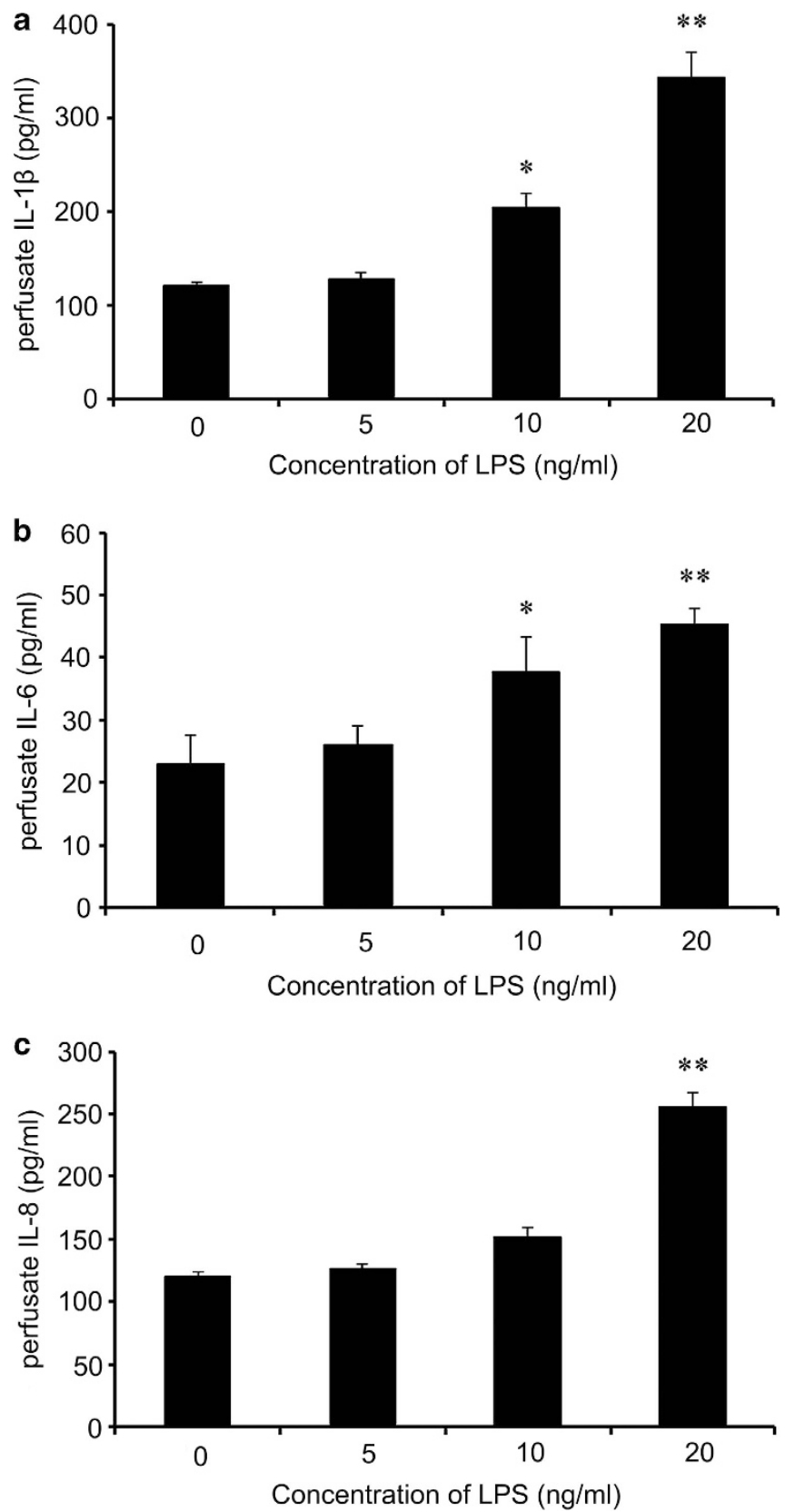

Figure 3 LPS induced the release of inflammatory cytokines in the superfusion system. The diseased periodontal tissue slices associated with the CS group were treated with various doses of LPS in a superfusion system, and then, the release of the inflammatory-related cytokines IL-1 $\beta$ (a), IL-6 (b) and IL-8 (c) was analyzed by ELISA. ${ }^{*} P<0.05,{ }^{*} P<0.01$. 
Therefore, we analyzed the protein levels of these two receptors in periodontal tissues with periodontitis with or without CS. As shown in Figure 2a, after periodontitis induction, the expression of $\alpha 1-\mathrm{AR}$ markedly increased in the periodontitis group (G2) (G1 versus G2; $P<0.01$ ), whereas the group with both periodontitis and CS (G3) exhibited even higher levels of $\alpha 1$ AR expression. However, the protein levels of $\beta 2$-AR were similar between G1 and G2 $(P>0.05)$ (Figure $2 \mathrm{~b})$ and only increased in G3. These protein expression data demonstrated that the inflammation (ligated sites) associated with CS resulted in significantly increased protein levels of both $\alpha 1$ $\mathrm{AR}$ and $\beta 2$-AR $(P<0.01)$ but that only the $\alpha 1$-AR response to inflammation was caused by simple periodontitis (G2). This suggests that $\alpha 1-\mathrm{AR}$ may have a more important role in the mechanisms underlying CS-accelerated periodontitis compared with $\beta 2$-AR.

LPS Simulated the inflammatory environment in vivo during periodontitis induction in a superfusion system

The superfusion system is a device that was developed by Raiteri's laboratory. ${ }^{21}$ It is suitable for a variety of applications involving the study of synaptic neurotransmitter release and the exploration of the physiological regulation and effect of agents that interfere with these actions. In this study, we used this system to simulate physiological processes in vivo. To promote the inflammatory environment in vivo during periodontitis induction, we first stimulated the G3 periodontal tissue slices with different concentrations of LPS to determine the appropriate concentration. Perfusate was collected, and the levels of the inflammatory-related cytokines IL-1 $\beta$, IL- 6 and IL-8 were quantified using an enzyme-linked immunosorbent assay kit.

After stimulation with different concentrations $(0,5,10$ and $20 \mathrm{ng} \mathrm{ml}^{-1}$ ) of LPS for $2 \mathrm{~h}$, a marked increase in IL-1 $\beta$ was observed compared with the control group, which reached a peak when the pretreatment was performed using $20 \mathrm{ng} \mathrm{ml}^{-1}$ LPS (Figure 3a). As shown in Figure 3b, preconditioning with $10 \mathrm{ng} \mathrm{ml}^{-1}$ LPS significantly increased the IL-6 levels, which were elevated by approximately twofold in the $20 \mathrm{ng} \mathrm{ml}^{-1}$ LPStreated groups. A similar situation occurred with the release of IL-8 (Figure 3c); when stimulated with $20 \mathrm{ng} \mathrm{ml}^{-1}$ LPS, a statistically significant difference was observed $(P<0.01)$. All of these results suggested that LPS at a concentration of 20 $\mathrm{ng} \mathrm{ml}{ }^{-1}$ could effectively simulate the inflammatory environment in vivo.

AR Blockade was responsible for the release of inflammatory cytokines in the superfusion system

It has been established that the ARs are targets of catecholamines, particularly norepinephrine (noradrenaline) and epinephrine (adrenaline). To determine whether AR activation mediates CS-enhanced periodontitis, the 1 l-AR blocker phentolamine $(50 \mu \mathrm{M})$ and $\beta 2$-AR blocker propranolol $(50 \mu \mathrm{M})$ were applied with $20 \mathrm{ng} \mathrm{ml}^{-1}$ LPS in the superfusion system as stimulatory factors for $2 \mathrm{~h}$. The enzyme-linked immunosorbent assay showed that there was a significant CS and phentolamine

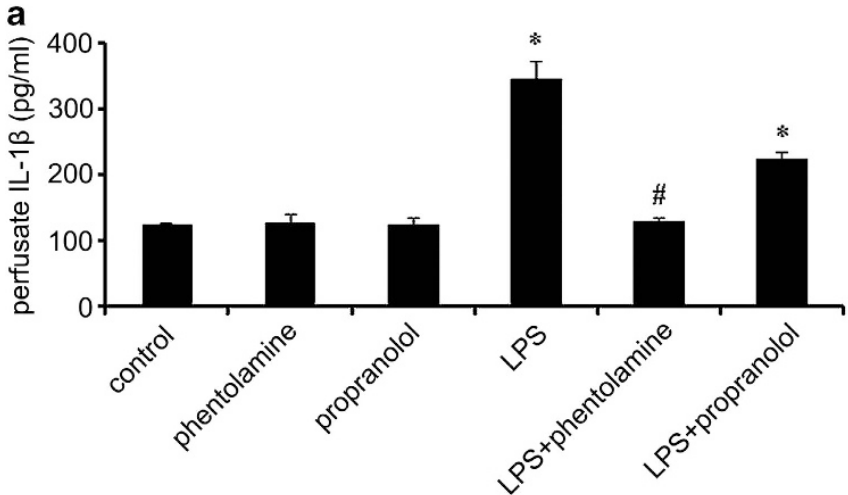

b
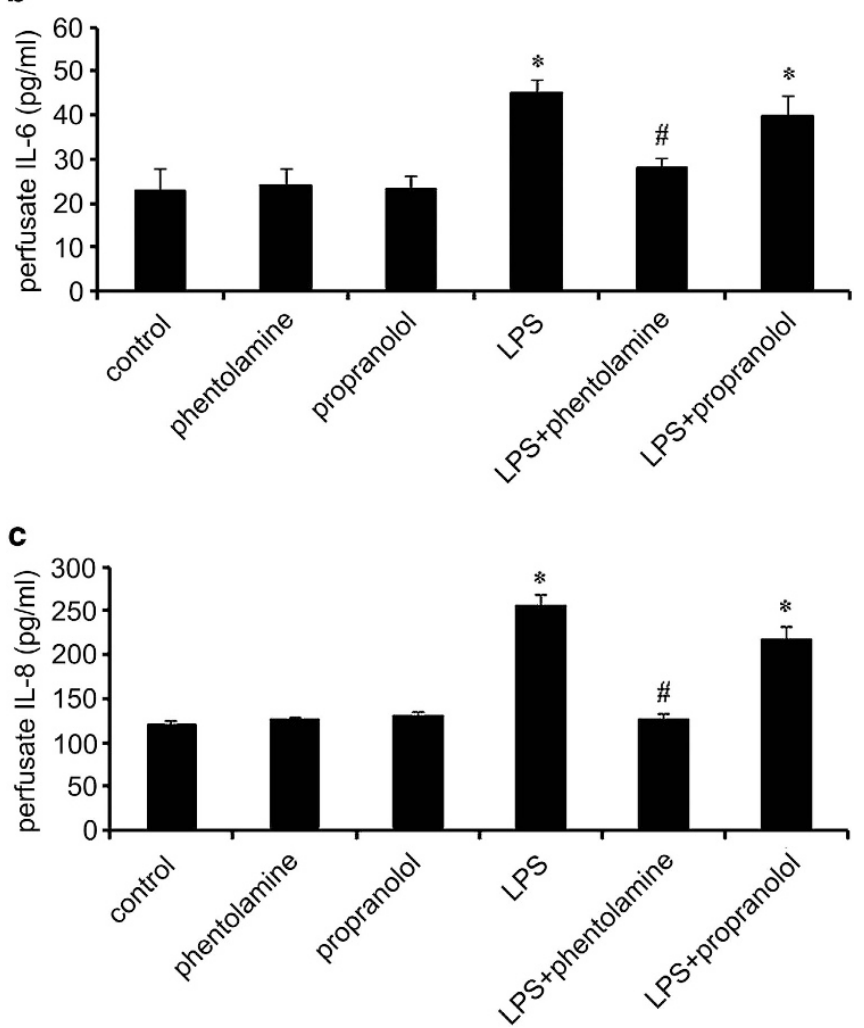

Figure 4 LPS induced the release of inflammatory cytokines mainly through $\alpha 1-A R$ signaling pathways. To clarify the underlying mechanisms, the AR blockers phentolamine and propranolol were introduced to block $\alpha 1-A R$ and $\beta 2$-ARsignaling. Following pretreatment with the AR blockers, the LPS-induced release of IL-1 $\beta$ (a), IL-6 (b) and IL-8 (c) was analyzed. *compared with control $P<0.05$, " compared with LPS $P<0.05$.

interaction effect on the inflammatory-related cytokines compared with the control group because the $\alpha 1$-AR blocker phentolamine significantly abrogated the CS-enhanced increases in IL-1 $\beta$ (Figure 4a), IL-6 (Figure 4b), and IL-8 (Figure $4 \mathrm{c}$ ) as induced by the LPS, whereas the $\beta 2$-AR blocker propranolol did not affect the cytokine levels. These results revealed that exposure to LPS in a CS environment similar to in vivo conditions triggered the release of IL-1 $\beta$, IL- 6 and IL-8, mainly through $\alpha 1$-adrenergic signaling pathways. 

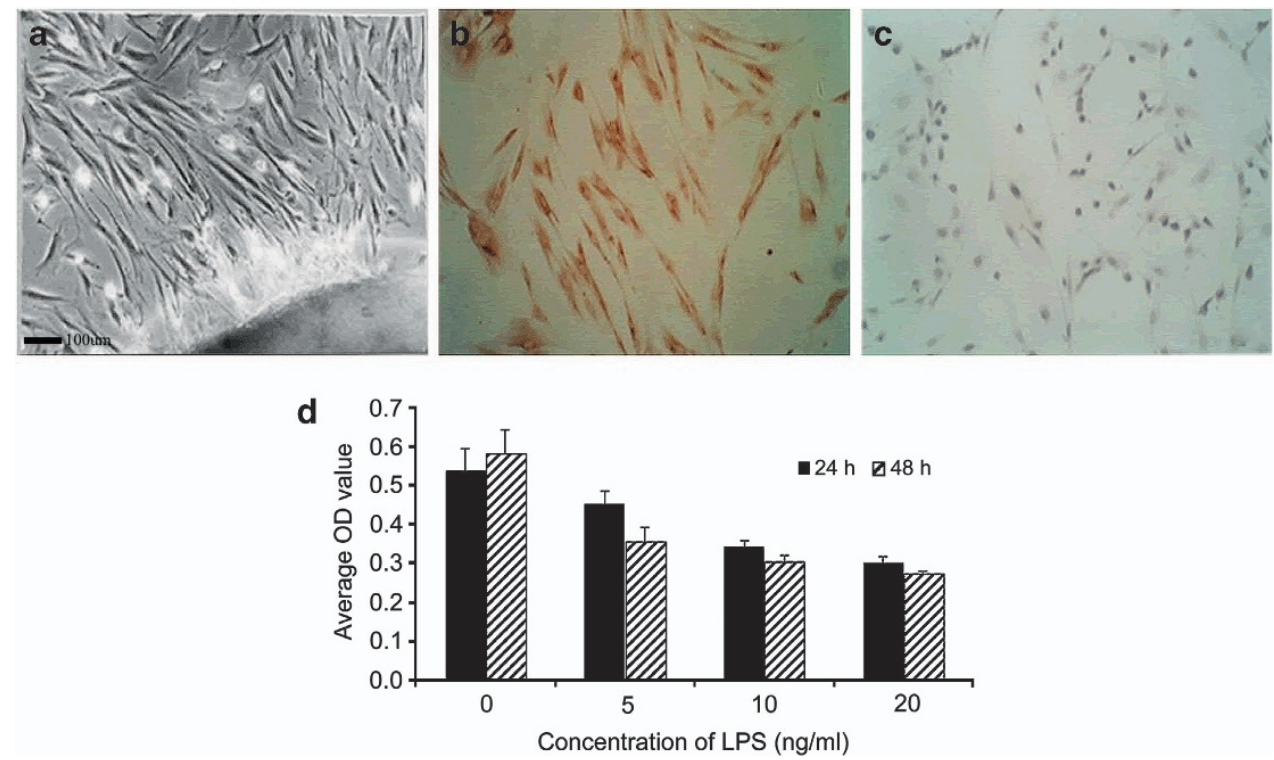

Figure 5 Characterization of HPDLFs by histological examinations with scanning electron microscopy (a). Immunohistochemistry analysis: vimentin-positive (b) and cytokeratin-negative (c) cells in the overlay were identified as differentiating HPDLFs. The HPDLFs were treated with various doses of LPS for $24 \mathrm{~h}$ or for $48 \mathrm{~h}$, and then, cell proliferation was analyzed by MTT assay (d). Scale bar $=100 \mu \mathrm{m}$.

\section{LPS-Inhibited HPDLF proliferation in a dose-dependent manner}

HPDLFs are the most important periodontal ligament cells that are involved in the synthesis and absorption of collagen and that can repair the periodontal ligament collagen to promote recovery from periodontitis. ${ }^{22}$ Our previous experiment confirmed that CS accelerated the inflammation of periodontitis; thus, we explored the effects of the ARs on the periodontal ligament cells under inflammatory conditions. As shown in Figures $5 \mathrm{a}-\mathrm{c}$, we observed large numbers of vimentin-positive and cytokeratin-negative cells, indicating that the HPDLFs had been successfully isolated.

To explore the effect of LPS on HPDLF proliferation, the 3-(4,5-dimethyl-2-thiazolyl)-2,5-diphenyl-2H-tetrazolium bromide assay was used. After stimulation with different concentrations of LPS for $24 \mathrm{~h}$ or for $48 \mathrm{~h}$, obvious decreases in the cell proliferation rates were observed compared with the control groups, which peaked following pretreatment with 20 $\mathrm{ng} \mathrm{ml}{ }^{-1}$ LPS for $48 \mathrm{~h}$ (Figure $5 \mathrm{~d}$ ). These results indicated that LPS restrained HPDLF proliferation in both dose-and timedependent manners.

\section{Treatments with AR blockers ameliorated LPS-suppressed HPDLF proliferation}

Reports have shown that increased surface AR expression is associated with enhanced sympathetic activity. ${ }^{23}$ To determine whether LPS enhance the sympathetic activities of HPDLFs by modulating AR expression, $20 \mathrm{ng} \mathrm{ml}^{-1}$ LPS were applied to the medium for $24 \mathrm{~h}$. Immunohistochemistry showed that LPS tended to increase the surface $\alpha 1-\mathrm{AR}$ expression compared with the control (Figure 6a), but no effects were observed on $\beta 2$-AR expression (Figure 6b), which is in accordance with the results of the aforementioned in vivo protein expression experiment. After preconditioning with the $\alpha 1$-AR blocker phentolamine, the proliferative abilities of the HPDLFs significantly increased compared with the LPS-treated group, indicating that the $\alpha 1-\mathrm{AR}$ blocker markedly ameliorated the LPS-inhibited cell proliferation (Figure 6c). However, the $\beta 2$-AR blockers did not abate the inhibitory effects of LPS on cell proliferation. Collectively, all of these results revealed that LPS triggered the extracellular proliferation of the HPDLFs, mainly through the $\alpha 1$-adrenergic signaling pathways.

\section{Effects of AR Blockade on LPS-induced release of inflammatory cytokines in vitro}

To investigate whether the AR blockade affected the LPSinduced release of inflammatory cytokines in the HPDLFs, the cells were stimulated with LPS followed by phentolamine or by propranolol for $24 \mathrm{~h}$. Then, the supernatants were collected, and the inflammatory-related cytokines IL-1 $\beta$, IL-6 and IL-8 were examined. The results showed that both phentolamine and propranolol reduced the IL- $1 \beta$ levels (Figure $7 \mathrm{a}$ ), but only phentolamine was able to reduce the IL-6 (Figure 7b) and IL-8 (Figure 7c) levels. As expected, $\alpha 1$-AR had an important role in the LPS-induced inflammation of the HPDLFs.

\section{DISCUSSION}

Periodontitis is a multifactorial disease that affects the periodontium, which encompasses the tissues surrounding and supporting the teeth. ${ }^{24}$ Periodontitis involves the progressive loss of the alveolar bones around the teeth, which leads to their loosening and subsequent loss. ${ }^{25}$ In recent studies, microorganisms have generally been considered to be the cause of periodontitis. ${ }^{26}$ However, it remains unclear whether bacteria are the only pathogens that cause periodontitis. Over the past decade, environmental risk factors for periodontitis have been 
a
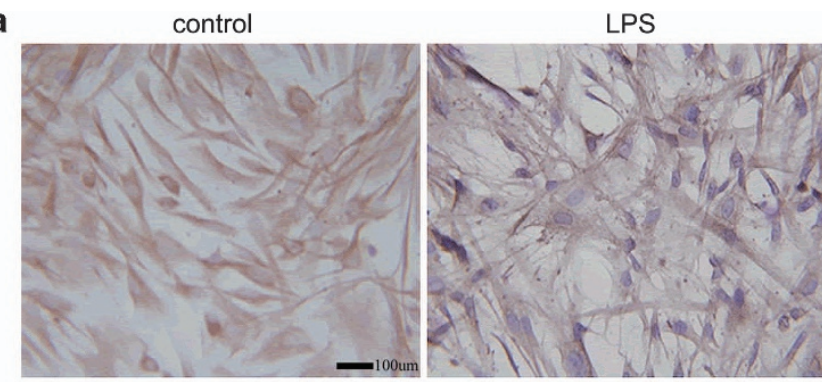

b

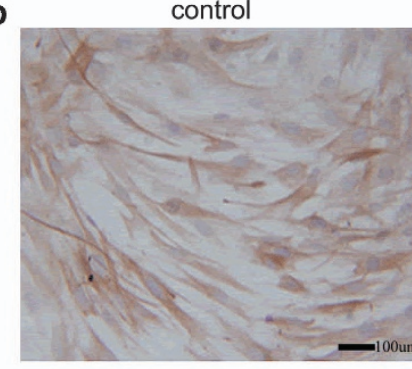

C

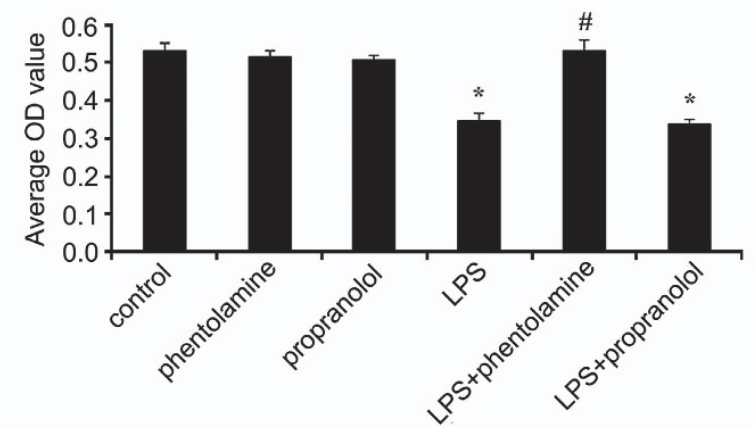

Figure 6 Effects of ARs on LPS-induced HPDLF proliferation. After stimulation with LPS for $24 \mathrm{~h}, \alpha 1-\mathrm{AR}$ (a) and $\beta 2-\mathrm{AR}$ (b) expression were observed by immunohistochemistry. Then, the AR blockers phentolamine and propranolol were introduced. HPDLF proliferation was also analyzed following the silencing of AR signaling (c). *compared with control $P<0.05$, " compared with LPS $P<0.05$. Scale bar $=100 \mu \mathrm{m}$.

extensively studied, and increasing evidence has emerged from epidemiologic studies relating this disease to stress, depression, and negative life events. ${ }^{27}$

Stress may be defined as a psycho-physiological response state of an organism facing the perception of a challenge or of a threat to the body, which can bring about physiological and metabolic perturbations. ${ }^{28}$ Stress promotes adaptation, but prolonged stress leads to sustained sympathetic activity levels, impaired immunity, atherosclerosis and the atrophy of nerve cells in the brain. ${ }^{29}$ Thus, stress is an important factor involved in the etiology and maintenance of many inflammatory diseases, including periodontal disease. ${ }^{30}$ Previous studies have suggested that CS causes a net negative effect on the immunological response of body, which leads to imbalances between hosts and parasites, resulting in further periodontal breakdown. ${ }^{27}$ Several clinical studies have investigated the possible relationship between psychological stress and periodontitis and have suggested that stress may have a role in the development of periodontal disease. ${ }^{30,31}$ However, the internal mechanisms responsible for the impacts of stress on the development of periodontitis remain unclear.

Because $\mathrm{TH}$ is the enzyme responsible for catalyzing the conversion of catecholamines to norepinephrine and epinephrine, the increased expression of $\mathrm{TH}$ most likely causes the increased activity of the sympathetic pathway. Therefore, in this study, we first assessed a rodent model of periodontitis using a nylon thread ligature placed around the neck of the maxillary first molar, which was also used in our previous study. Then, multiple stimulations of CS were provided. As the experiment progressed, more severe inflammatory changes were observed in association with the periodontitis of the CS group compared with the simple periodontitis group. At the end of the 30-day experimental period, tissue blocks from the maxilla were collected for further analysis. Diagnoses of periodontitis established by the amounts of bone loss around the teeth demonstrated that CS was able to dramatically promote the progression of this disease. In addition, the pretreatment with CS was able to significantly enhance TH expression levels in the three groups, indicating that CS may accelerate the pathological process of periodontitis via the sympathetic pathway.

When the important sympathetic neurotransmitter catecholamine is converted to epinephrine, it can be captured by ARs on the cell surface and exert biological effects. Among all of the ARs, $\alpha 1-A R$ and $\beta 2-A R$ are generally considered to be involved in brain-immune communication. Therefore, we assessed both $\alpha 1-\mathrm{AR}$ and $\beta 2-\mathrm{AR}$ in this study. As expected, the $\alpha 1-\mathrm{AR}$ and $\beta 2$-AR protein expression significantly increased following the CS treatment, but only the $\alpha 1-\mathrm{AR}$ levels were responsive in the periodontitis group. We then investigated whether ARs mediate the underlying mechanisms of the CS-accelerated pathological processes of periodontitis and the roles of these two receptors in the associated molecular signal transduction pathways. We used the superfusion system with LPS to simulate in vivo inflammatory reactions. The $\alpha 1$-AR blocker phentolamine and the $\beta 2$-AR blocker propranolol were applied with the stimulatory LPS to the periodontal tissues of the CS-treated periodontitis rats. The inflammatory cytokine levels of IL-1 $\beta$, IL- 6 and IL-8 were expected to decrease following the $\alpha 1-\mathrm{AR}$ blocker phentolamine pretreatment in the LPS-treated group. However, the $\beta 2$-AR blocker propranolol did not greatly affect the LPS-induced cytokine levels. Therefore, these results confirmed that CS-accelerated periodontitis progresses mainly through the $\alpha 1$-adrenergic signaling pathway.

HPDLFs are the most important periodontal ligament cells involved in the synthesis and absorption of collagen. They can repair the periodontal ligament collagen and aid in recovery from periodontitis. Our previous experiment confirmed that pretreatment with CS could accelerate the inflammatory response associated with periodontitis; therefore, to further investigate the mechanisms involved in CS-accelerated periodontitis, we used LPS to simulate the inflammatory environment to explore the manner by which inflammatory processes impact HPDLFs in vitro. As expected, in the LPS-simulated inflammatory environment, the proliferation of the HPDLFs 

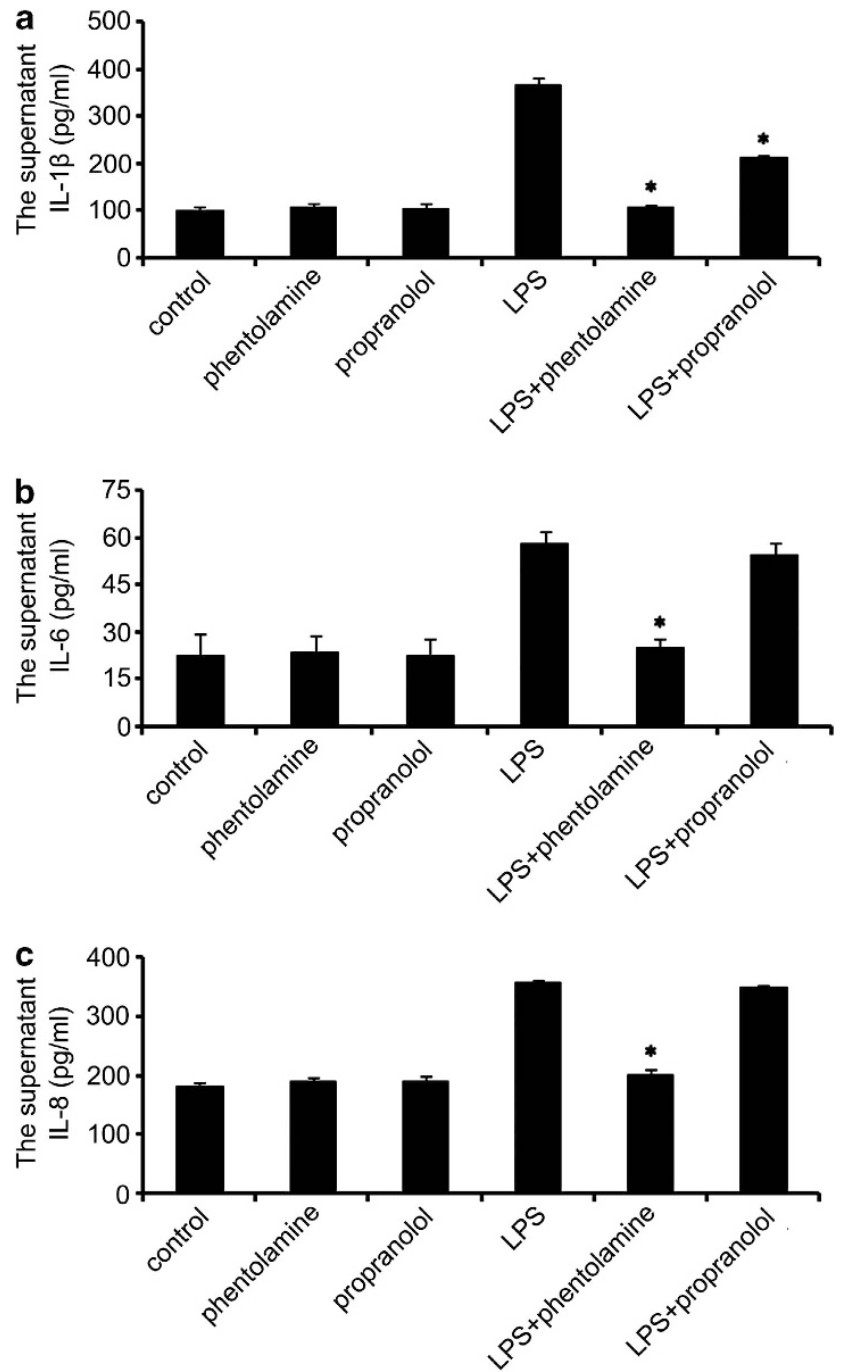

Figure 7 Effects of AR blockade on LPS-induced release of inflammatory cytokines in vitro. Following pretreatment with $25 \mu \mathrm{M}$ AR blocker for $24 \mathrm{~h}$, the supernatant was collected, and IL-1 $\beta$ (a), IL-6 (b) and IL-8 (c) levels were examined. *compared with LPS $P<0.05$.

markedly decreased and the release of the inflammatory cytokines significantly increased compared with the control group, similar to the pattern of $\alpha 1-\mathrm{AR}$ expression observed. Interestingly, phentolamine administration significantly attenuated the effects of the LPS-induced decrease in proliferation and increased secretion of IL-1 $\beta$, IL-6, IL-8. These effects were not apparent in the propranolol-treated group, however. Thus, our data confirmed that CS-accelerated periodontitis progressed through the $\alpha 1$-adrenergic signaling pathways.

In this study, we have shown that CS accelerates the progression of periodontitis by exciting the sympathetic nervous system, resulting in the release of the catecholamine neurotransmitter epinephrine, which is able to bind to the $\alpha 1-\mathrm{AR}$ on the periodontal cell surface, reducing the biological activities of the cells and causing the massive release of inflammation-related factors. Thus, our study provides novel insight into the manner by which CS accelerates the pathological process of periodontitis and may facilitate the development of a therapeutic strategy for periodontal disease.

\section{CONFLICT OF INTEREST}

The authors declare no conflict of interest.

\section{ACKNOWLEDGEMENTS}

This work was supported by the National Natural Science Foundation of China (81070837).

1 Darveau RP. Periodontitis: a polymicrobial disruption of host homeostasis. Nat Rev Microbiol 2010; 8: 481-490.

2 Peruzzo DC, Benatti BB, Antunes IB, Andersen ML, Sallum EA, Casati MZ et al. Chronic stress may modulate periodontal disease: a study in rats. J Periodontol 2008; 79: 697-704.

3 Zarco M, Vess T, Ginsburg G. The oral microbiome in health and disease and the potential impact on personalized dental medicine. Oral Dis 2012; 18: 109-120.

4 Deo V, Bhongade M. Pathogenesis of periodontitis: role of cytokines in host response. Dentistry today 2010; 29: 60-68.

5 Gašperšič R, Štiblar-Martinčič D, Skalerič U. Influence of restraint stress on ligature-induced periodontitis in rats. Eur J Oral Sci 2002; 110: $125-129$.

6 Dhabhar FS. Enhancing versus suppressive effects of stress on immune function: Implications for immunoprotection and immunopathology. Neuroimmunomodulation 2009; 16: 300-317.

7 Genco RJ, Borgnakke WS. Risk factors for periodontal disease. Periodontol 2000 2013; 62: 59-94.

8 Di Loreto DJ. Comparing Stress Responses in Generalized Anxiety Disorder vs. Non-Clinical Populations: a Cortisol and Alpha-Amylase Study. University of Tennessee, 2013.

9 Katori Y, Shibata S, Kawase T, Cho BH, Murakami G. Transient appearance of tyrosine hydroxylase immunoreactive cells in the midline epithelial seam of the human fetal secondary palate. Cleft Palate Craniofac J 2012; 49: 414-424.

10 Venkatakrishnan A, Deupi X, Lebon G, Tate CG, Schertler GF, Babu MM. Molecular signatures of g-protein-coupled receptors. Nature 2013; 494: 185-194.

11 Rasmussen SG, DeVree BT, Zou Y, Kruse AC, Chung KY, Kobilka TS et al. Crystal structure of the [bgr] 2 adrenergic receptor-gs protein complex. Nature 2011; 477: 549-555.

12 Siniscalchi M, Sasso R, Pepe AM, Dimatteo S, Vallortigara G, Quaranta A Catecholamine plasma levels following immune stimulation with rabies vaccine in dogs selected for their paw preferences. Neurosci Lett 2010; 476: 142-145.

13 Marvar PJ, Lob H, Vinh A, Zarreen F, Harrison DG. The central nervous system and inflammation in hypertension. Curr Opin Pharmacol 2011; 11: 156-161.

14 Carvalho RS, de Souza CM, Neves JC, Holanda-Pinto SA, Pinto LM, Brito GA et al. Research effect of venlafaxine on bone loss associated with ligature-induced periodontitis in wistar rats. BioMed 2010; 9: 3-11.

15 Kayal RA. The role of osteoimmunology in periodontal disease. Biomed Res Int 2013; 2013: e12

16 Willner P, Muscat R, Papp M. Chronic mild stress-induced anhedonia: A realistic animal model of depression. Neurosci Biobehavl $R$ 1992; 16: 525-534.

17 Lamkin DM, Sloan EK, Patel AJ, Chiang BS, Pimentel MA, Ma JC et al. Chronic stress enhances progression of acute lymphoblastic leukemia via $\beta$-adrenergic signaling. Brain Behav Immun 2012; 26: 635-641.

18 Meister A. Advances in Enzymology and Related Areas of Molecular Biology. John Wiley \& Sons: NY, USA, 2009.

19 Nagatsu T. Tyrosine hydroxylase: Human isoforms, structure and regulation in physiology and pathology. Essays Biochem 1995; 30: 15-35. 
20 Cortez V, Santana M, Marques AP, Mota A, Rosmaninho-Salgado J, Cavadas C. Regulation of catecholamine release in human adrenal chromaffin cells by $\beta$-adrenoceptors. Neurochem Int 2012; 60: 387-393.

21 Romei C, Raiteri M, Raiteri L. Gaba transporters mediate glycine release from cerebellum nerve endings: Roles of ca 2+ channels, mitochondrial na/ ca $2+$ exchangers, vesicular gaba/glycine transporters and anion channels. Neurochem Int 2012; 61: 133-140.

22 El-Awady AR, Messer RL, Gamal AY, Sharawy MM, Wenger KH, Lapp CA. Periodontal ligament fibroblasts sustain destructive immune modulators of chronic periodontitis. J Periodontol 2010; 81: 1324-1335.

23 DeLorey DS, Clifford PS, Mittelstadt S, Anton MM, Kluess HA, Tune JD et al. The effect of aging on adrenergic and nonadrenergic receptor expression and responsiveness in canine skeletal muscle. J App/ Physiol 2012; 112: 841-848.

24 Savage A, Eaton KA, Moles DR, Needleman I. A systematic review of definitions of periodontitis and methods that have been used to identify this disease. J Clin Periodontol 2009; 36: 458-467.

25 Slots J, Ting M. Actinobacillus actinomycetemcomitans and porphyromonas gingivalis in human periodontal disease: occurrence and treatment. Periodontol 2000 1999; 20: 82-121.

26 Inaba H, Amano A. Roles of oral bacteria in cardiovascular diseases-from molecular mechanisms to clinical cases: Implication of periodontal diseases in development of systemic diseases. J Pharmacol Sci 2010; 113: 103-109.

27 Rai B, Kaur J, Anand S, Jacobs R. Salivary stress markers, stress, and periodontitis: a pilot study. J Periodontol 2011; 82: 287-292.
28 Rasmusson AM, Schnurr PP, Zukowska Z, Scioli E, Forman DE. Adaptation to extreme stress: Post-traumatic stress disorder, neuropeptide y and metabolic syndrome. Exp Biol Med 2010; 235: 1150-1162.

29 McEwen BS, Gianaros PJ. Central role of the brain in stress and adaptation: Links to socioeconomic status, health, and disease. Ann NY Acad Sci 2010; 1186: 190-222.

30 Akcali A, Huck O, Tenenbaum H, Davideau J, Buduneli N. Periodontal diseases and stress: a brief review. J Oral Rehabil 2013; 40: 60-68.

31 Stabholz A, Soskolne WA, Shapira L. Genetic and environmental risk factors for chronic periodontitis and aggressive periodontitis. Periodontol 2000 2010; 53: 138-153.

(c) (1) () $(-$ This work is licensed under a Creative Commons Attribution-NonCommercial-NoDerivs 3.0 Unported License. The images or other third party material in this article are included in the article's Creative Commons license, unless indicated otherwise in the credit line; if the material is not included under the Creative Commons license, users will need to obtain permission from the license holder to reproduce the material. To view a copy of this license, visit http://creativecommons.org/licenses/by-nc-nd/3.0/ 